\title{
Brain Disorders in Critical Illness: Mechanisms, Diagnosis, and Treatment
}

\author{
Robert D. Stevens, Tarek Sharshar, E. Wesley Ely (Editors). Cambridge University Press, \\ \$102.83. 2013, ISBN 978-1-107-02919-4
}

\author{
Jason Waechter, MD
}

Received: 10 April 2014/ Accepted: 23 April 2014/Published online: 11 June 2014

(C) Canadian Anesthesiologists' Society 2014

This 440-page book is organized into six sections: Epidemiology and Outcomes, Behavioral Neurology in the ICU, Biological Mechanisms, Diagnosis of Brain Dysfunction, Preventative and Therapeutic Interventions, and Clinical Encephalopathy Syndromes. A quick scan of the chapter titles reveals four chapters on sepsis, five on delirium, five related to pharmacology, and three on ischemia/reperfusion, plus chapters on other assorted topics.

With respect to organization, each chapter is six to ten pages in length and can be read easily in one sitting. Furthermore, the chapters are uniformly organized with an initial summary, introduction, body, and conclusion that facilitate readability. I appreciate the editors' efforts to ensure consistency of these features across all chapters. General subject areas are clustered together by section, but some topics are also dispersed throughout the book. For example, chapters on delirium are scattered among four different sections in the book, yet I was able to locate them easily.

From my personal clinical perspective (including working in multi-systems, neurotrauma, and post-cardiac surgery intensive care units [ICU]), delirium, sepsis, trauma, and cerebrovascular events comprise a majority of brain pathology and dysfunction in the ICU - three of these four topics are well represented in the chapter titles.

Some of the topics in the section on Behavior Neurology in the ICU represent relatively novel content, e.g., the chapter on Neural Basis of Fear and Anxiety, and I value the inclusion of these topics given the increasing awareness

J. Waechter, MD $(\bowtie)$

Departments of Critical Care and Anesthesiology, University

of Calgary, Calgary, AB, Canada

e-mail: jwaech@yahoo.ca of ICU post-traumatic stress disorder. All chapters in this section are likely beyond the personal knowledge level of most intensivists and thus represent good learning opportunities. There is also a strong psychology influence in many of the chapters.

In the section on Biological Mechanisms, there is an emphasis on neuroanatomy, neuropsychology, and neurobiochemistry. This well-referenced and supported text leaves the reader with few stones unturned. A challenge in having multiple authors is striking a balance between chapter coherence and redundancy. In this case, the editors have effectively ensured that chapters with common themes (e.g., delirium and sepsis) maintain their focus on the discrete components without covering the basics again and again. The chapter on Neurobiological Effects of Systemic Physiological and Metabolic Insults provides a useful overview of the pathophysiology and interpretation of cerebral microdialysis, which is increasingly being seen in the realm of neurointensive care.

The section on Diagnosis of Brain Dysfunction is more clinically oriented than the previous sections. It begins appropriately with a clinical neurologic assessment of the critically ill patient and is well organized into tables for brainstem reflexes, National Institutes of Health stroke scale, Glasgow Coma Scale, and aphasia categories. I looked for inclusion of other physical findings in this chapter, such as asterixis, posturing, clonus, diaphoresis, autonomic lability, breathing patterns, meningeal signs, skin findings, and odours (ketones), but I did not find them. Additional physical findings, such as distended bladder in a delirious patient, are not necessarily central to a neurologic exam, but they are essential in the overall assessment of the patient and worthy of mention. As another example, Table 23.1 in this chapter provides a comprehensive list of etiologies of acute weakness; a translation of this list to 
the physical exam portion of the clinical assessment would be a valued addition. Perhaps an approach to clinical neurological syndromes (e.g., weakness, coma, or seizure) would be useful. I would expect the level of detail in this section to match the level of detail in the previous section on Biological Mechanisms, but this wasn't the case.

The following chapter on Bedside Assessment of Delirium in critically ill patients provides extensive detail on the assessment of scoring systems. An assessment into the factors that contribute to delirium, e.g., pain, was given mention; however, assessments of sleep, physical mobility, ventilator comfort, new infections, and a search for other physiological causes of delirium were not included. Delirium assessment, which includes the initial detection of delirium, is well represented in this chapter by the delirium scoring assessment, but an assessment to determine causative factors, also an important component of the assessment, is lacking in this chapter. An entire chapter on the pharmacotherapy of delirium is included in the text, but an increased focus on non-pharmacotherapy could have been equally emphasized.

The section on Clinical Encephalopathy Syndromes provides concise reviews of each of the syndromes described, all in a consistent format. The topics are up to date and relevant. Elevated intracranial pressure (ICP) is a common abnormality in neurocritical care and can manifest as encephalopathy. As well, there are many considerations of treatment of subarachnoid hemorrhage, including encephalopathy caused by vasospasm, elevated ICP, obstructive hydrocephalus, and ventriculitis. These common neurocritical care illnesses could have been more deserving of attention. A dedicated chapter on brain death could also have been an important inclusion.

Overall, from a clinical perspective, the chapters are concise, well written, and well organized, but there are some areas of important content which, in my view, are valuable additions which should have been included. This very strong and well-referenced basic science text covers a broad range of topics including neuroanatomy, neurophysiology, and biochemistry, and delivers a solid foundation that clinicians can translate to an increased understanding of brain dysfunction at bedside. I would recommend this book for ICU physicians with clinical and research interests in neurocritical care.

Conflict of interest None declared. 